\title{
Early diagnosis of bladder cancer through the detection of urinary tyrosine-phosphorylated proteins
}

\begin{abstract}
A Khadjavi ${ }^{1}$, F Mannu ${ }^{2}$, P Destefanis ${ }^{3}$, C Sacerdote $^{4}$, A Battaglia $^{3}$, M Allasia ${ }^{3}$, D Fontana $^{3}$, B Frea $^{3}$, S Polidoro $^{5}$, G Fiorito ${ }^{5}$, G Matullo ${ }^{5}$, A Pantaleo ${ }^{6}$, A Notarpietro ${ }^{7}$, M Prato ${ }^{1}$, F Castagno ${ }^{8}$, P Vineis ${ }^{9}$, P Gontero ${ }^{3}$, G Giribaldi ${ }^{*, 7,10}$ and F Turrini ${ }^{7,10}$

${ }^{1}$ Department of Neurosciences, University of Turin, Torino, Italy; ${ }^{2}$ Nurex s.r.l., Sassari, Italy; ${ }^{3}$ Urology Clinic, Città della Salute e della Scienza di Torino, Hospital San Giovanni Battista, Department of Surgical Sciences, University of Turin, Torino, Italy; ${ }^{4}$ Unit of Cancer Epidemiology, Department of Medical Sciences, University of Turin and Centre for Cancer Epidemiology and Prevention (CPO Piemonte), Torino, Italy; ${ }^{5} \mathrm{HuGeF}$ Human Genetics Foundation and Department of Medical Sciences, University of Turin, Torino, Italy; ${ }^{6}$ Department of Biomedical Sciences, University of Sassari, Sassari, Italy; ${ }^{7}$ Department of Oncology, University of Turin Medical School, University of Turin, via Santena 5 BIS, Torino 10126, Italy; ${ }^{8}$ Blood Center, AO Città della Salute e della Scienza di Torino, Hospital San Giovanni Battista, Torino, Italy and ${ }^{9}$ MRC-PHE Center for Environment and Health, School of Public Health, Imperial College, London, UK
\end{abstract}

Background: A noninvasive, highly sensitive and specific urine test is needed for bladder cancer (BC) diagnosis and surveillance in addition to the invasive cystoscopy. We previously described the diagnostic effectiveness of urinary tyrosine-phosphorylated proteins (UPY) and a new assay (UPY-A) for their measurement in a pilot study. The aim of this work was to evaluate the performances of the UPY-A using an independent cohort of 262 subjects.

Methods: Urinary tyrosine-phosphorylated proteins were measured by UPY-A test. The area under ROC curve, cutoff, sensitivity, specificity and predictive values of UPY-A were determined. The association of UPY levels with tumour staging, grading, recurrence and progression risk was analysed by Kruskal-Wallis and Wilcoxon's test. To test the probability to be a case if positive at the UPY-A, a logistic test adjusted for possible confounding factor was used.

Results: Results showed a significant difference of UPY levels between patients with BC vs healthy controls. For the best cutoff value, 261.26 Standard Units (SU), the sensitivity of the assay was $80.43 \%$ and the specificity was $78.82 \%$. A statistically significant difference was found in the levels of UPY at different BC stages and grades between Ta and T1 and with different risk of recurrence and progression. A statistically significant increased risk for BC at UPY-A $\geqslant 261.26$ SU was observed.

Conclusions: The present study supplies important information on the diagnostic characteristics of UPY-A revealing remarkable performances for early stages and allowing its potential use for different applications encompassing the screening of high-risk subjects, primary diagnosis and posttreatment surveillance.

Urinary bladder cancer (BC) ranks ninth in cancer incidence worldwide (Ploeg et al, 2009; Chavan et al, 2014). The diagnosis is made after symptom observation and urethrocystoscopy (UCS)
(Boman et al, 2002; Babjuk et al, 2014). More than 50\% of nonmuscle-invasive (NMIBC: CIS, Ta, T1) patients experienced at least one recurrence, whereas $10-15 \%$ have a progression to an

\footnotetext{
*Correspondence: Dr G Giribaldi; E-mail: giuliana.giribaldi@unito.it

${ }^{10}$ These authors contributed equally to this work.
}

Received 27 January 2015; revised 13 May 2015; accepted 26 May 2015; published online 30 June 2015

(c) 2015 Cancer Research UK. All rights reserved 0007-0920/15 
invasive form (Simon et al, 2003). Patients undergo a lifelong follow-up, and also for this reason $\mathrm{BC}$ is the most costly cancer from diagnosis to death (Hong and Loughlin, 2008; Gore and Gilbert, 2013).

Several markers have been proposed, but none of them was able to replace the UCS in the diagnosis and follow-up of BC (Cheung et al, 2013) as documented by the current urological guidelines (AUA, EAU and NCCN). It should be noted that estimates of UCS false negative range from 10 to $40 \%$ (Kriegmair et al, 1996; Schneeweiss et al, 1999; Zaak et al, 2001), and specificity can be as low as $37 \%$ (Sarosdy et al, 2002). Although available urine markers are not usually considered to possess sufficient sensitivity and specificity for the screening of BC in the general population (Parker and Spiess, 2011; Cheung et al, 2013), many markers have shown potential value in improving diagnostic accuracy when used to complement current strategies or when multiple markers are used (Miremami and Kyprianou, 2014). Nevertheless, it should be noted that some markers with comparable performances are used for the screening of other tumours (Greene et al, 2013). As a possible cause of their limited use, the urinary tests showing better diagnostic performances such as uCyt + /immunoCyt, microsatellite and FISH require personnels with specific training, have low throughput and are very expensive (Cheung et al, 2013). Therefore, to be widely usable and to allow a wide clinical validation, new urinary tests for $\mathrm{BC}$ should be standardised, easier to interpret and cost-effective (Cheung et al, 2013).

The present study grounds on our previous proteomic analyses of $\mathrm{BC}$ tissue and urine revealing the presence of anomalous levels of tyrosine-phosphorylated proteins (Khadjavi et al, 2011). Urinary tyrosine-phosphorylated proteins (UPY) showed remarkable stability in urine but their low concentration initially required complex and expensive proteomic techniques for their measurement (Khadjavi et al, 2011), thus limiting their practical utility. Substantial work was then required to miniaturise the method encompassing all purification and detection steps, to standardise the results and to limit the assay costs. Its performances were investigated in a training set of subjects (Khadjavi et al, 2013). Therefore, the aim of the present study was to evaluate the UPY assay (UPY-A) in an independent set of subjects and its diagnostic performances at different tumour stages and grades, its association with tumour progression and recurrence risk and the effects of possible confounding factors such as age, smoking status and gender.

\section{MATERIALS AND METHODS}

Patients and sample collection. Urine samples from newly diagnosed BC patients were collected at the Urology Clinic of Città della Salute e della Scienza, Hospital San Giovanni Battista, University of Turin. Urine samples from healthy volunteers were obtained from the Blood Bank of the same hospital. Patients with suspected BC were enrolled in this study before undergoing transurethral resection of the bladder. Patients with a histological diagnosis (reference standard) different from $\mathrm{BC}$ or with a previous BC history were subsequently excluded. The list of recruited patients included people who: (1) received a first diagnosis of BC between September 2010 to May 2012; (2) lived in the study area at the time of diagnosis; (3) were over age 18 years; (4) were able to provide interview data. Healthy controls included people who did not present symptoms or signs of $\mathrm{BC}$, previous $\mathrm{BC}$ history and meeting the criteria 2,3 and 4. All recruited subjects underwent the test. The study was approved by the local research Ethical Committee and was conducted according to Declaration of Helsinki's prescriptions. All the subjects included in the protocol signed a declaration of informed consent and received a brief questionnaire covering detailed medical and personal information.
The subjects were classified as 'current smokers', 'former smokers' (quit smoking for at least 10 years) and 'nonsmokers'. A total of 260 participants provided age information, 239 smoking status and 262 gender information. Bladder cancer grade and stage were determined according to the WHO (1973 and 2004) criteria and TNM classification, respectively. Risk scores for recurrence and progression were calculated for each patient affected by NMIBC according to the EORTC definition. These factors comprise tumour grade, stage, size, number and concomitant CIS. Based on these scores, patients were considered to have very low (score 0 ), low (score 1-4), moderate (scores 5-9) or high (scores 10-17) risk for recurrence and very low (score 0), low (scores 2-6), moderate (scores 7-13) or high (scores 14-23) risk for progression (Babjuc et al, 2014). Voided urine samples (10-50 ml) were collected from the second micturition of the morning. Samples were stored at $-20^{\circ} \mathrm{C}$ within $2 \mathrm{~h}$ from collection. The test was performed within 6 months from collection. No significant decay of UPY levels have been noticed after 2 years of cold storage.

Measurement of urine tyrosine-phosphorylated proteins. Urine samples were centrifuged for $20 \mathrm{~min}$ at $700 \mathrm{~g}$ at $10^{\circ} \mathrm{C}$ and supernatants were collected. Five hundred microlitres of supernatant from each patient were processed using the UPY-A (Khadjavi et al, 2013). Detection was performed by using a standard chemiluminescence reader (Synergy HT Multi-Mode Microplate Reader; Biotek, AHSI, Milano, Italy): luminescence end point, sensitivity $100 \%$ and integration time $1.0 \mathrm{~s}$. Using an external peptide calibration curve, UPY levels were interpolated and expressed as Standard Units (SU).

Statistical analyses. Summary data are presented as means, medians and standard deviations for continuous variables and as percentages for categorical variables. Differences between BC cases and healthy controls were tested using the nonparametric Wilcoxon's rank-sum test or $\chi^{2}$ test for continuous variables or categorical variables, respectively. The accuracy of the UPY-A biomarker was tested computing the area under the ROC curve (AUC). Different cutoff levels were used to determine which performs better. Positive predictive value (PPV) and negative predictive value (NPV) were also computed at each cutoff point. The Kruskal-Wallis test was used to assess if UPY levels were different between groups characterised by different tumour stage and grade, as well as different recurrence or progression risks. The increase in the prediction performance in predicting recurrence and progression given by the UPY-A marker with respect to the EORTC risk class was evaluated computing the AUC of three logistic models, including age, gender, smoke: model 1, + EORTC risk class; model 2, + UPY-A marker; model 3, + UPY-A marker + EORTC risk class. The AUC of the three models were compared by means of the DeLong test (DeLong et al, 1988). To test the probability to be a case if positive at the UPY-A, we used a multivariate logistic regression adjusted for age, smoking status and gender. All tests were two-sided and we considered a 5\% significance level. Analyses were performed using SAS software, version 9.2 (SAS Institute Inc., Milano, Italy).

\section{RESULTS}

Evaluation and optimisation of urine tyrosine-phosphorylated protein assay. To evaluate the results concerning UPY obtained in the pilot study (Khadjavi et al, 2013) in an independent cohort of subjects, 262 new urinary samples collected from 92 BC patients and 170 healthy subjects were analysed (Table 1 ). Urinary tyrosinephosphorylated protein levels showed a significant difference $\left(P=1.71 \times 10^{-23}\right.$ Wilcoxon's rank-sum test $)$ between patients with BC vs healthy controls (means: $434.8 \pm 258.4$ vs $157.9 \pm 114.6 \mathrm{SU}$ ), corresponding to an $\sim 4$-fold increase of UPY 
Table 1. Clinical and pathologic characteristics of subjects

\begin{tabular}{|c|c|c|c|c|}
\hline & \multicolumn{2}{|c|}{ Controls } & \multicolumn{2}{|c|}{ Bladder cancer cases } \\
\hline & No. & $\%$ & No. & $\%$ \\
\hline Total $(n=262)$ & 170 & 65 & 92 & 35 \\
\hline \multicolumn{5}{|l|}{ Sex } \\
\hline Women & 39 & 15 & 6 & 2 \\
\hline Man & 131 & 50 & 86 & 33 \\
\hline \multicolumn{5}{|l|}{ Age (years) } \\
\hline$\leqslant 55$ & 53 & 20 & 11 & 4 \\
\hline $56-65$ & 72 & 28 & 25 & 9 \\
\hline $66-75$ & 28 & 11 & 37 & 14 \\
\hline$>75$ & 15 & 6 & 19 & 7 \\
\hline Missing & 2 & 1 & & \\
\hline \multicolumn{5}{|l|}{ Smoking } \\
\hline Current smokers & 28 & 11 & 53 & 20 \\
\hline Former smokers & 10 & 4 & 20 & 8 \\
\hline Non-smokers & 111 & 42 & 17 & 6 \\
\hline Missing & 21 & 8 & 2 & 1 \\
\hline \multicolumn{5}{|c|}{ Histology at first diagnosis } \\
\hline $\mathrm{CIS}$ & & & 4 & 4 \\
\hline Ta & & & 53 & 53 \\
\hline T1 & & & 20 & 22 \\
\hline T2-3 & & & 15 & 16 \\
\hline \multicolumn{5}{|c|}{ Grading at first diagnosis } \\
\hline $\mathrm{CIS}$ & & & 4 & 4 \\
\hline 1 & & & 29 & 33 \\
\hline 2 & & & 29 & 33 \\
\hline 3 & & & 30 & 34 \\
\hline \multicolumn{5}{|l|}{ WHO 2004} \\
\hline Low grade & & & 43 & 47 \\
\hline High grade & & & 43 & 47 \\
\hline Missing & & & 6 & 6 \\
\hline \multicolumn{5}{|c|}{ Risk of recurrence $(n=73)$} \\
\hline Very low & & & 24 & 33 \\
\hline Low & & & 40 & 55 \\
\hline Moderate & & & 9 & 12 \\
\hline \multicolumn{5}{|c|}{ Risk of progression $(n=73)$} \\
\hline Very low & & & 40 & 55 \\
\hline Low & & & 17 & 23 \\
\hline Moderate & & & 16 & 22 \\
\hline
\end{tabular}

(Figure 1A). We performed the ROC curve analysis (Figure 1B), and the ROC AUC including UPY-A, age, smoking status and gender as predictors was 0.92 , with a $95 \%$ confidence interval (CI) of $0.89-0.97$. For the best cutoff value (261.26 SU), the performances of the UPY-A, using only the test as a predictor, were calculated, displaying a sensitivity of $80.43 \%$ and a specificity of $78.82 \%$, with a PPV of $67.3 \%$ and an NPV of $88.2 \%$. It should be noted that the sensitivity of the assay is still $57.61 \%$, with $95.29 \%$ of specificity. To evaluate possible interferences, we tested 16 urine samples from patients with cystitis and variable levels of leukocyturia and hematuria. The obtained results (mean values: $179.1 \pm 117.1 \mathrm{SU})$ were not significantly different from control subjects ( $P=0.364$ Wilcoxon's rank-sum test).

Diagnostic performances of urine tyrosine-phosphorylated protein assay at different stages and grades of BC. The diagnostic performances of the assay have been evaluated according to tumour stages and grades. Figure 2A shows the levels of UPY-A in control, CIS, Ta, T1, T2-3 tumour stages, whereas Figure $2 \mathrm{~B}$ shows the variations observed in G1, G2 and G3. A statistically significant difference was found in UPY levels between the control group and the groups of patients at different tumour stages and grades $\left(P=8.10 \times 10^{-22}\right.$ and $P=6.99 \times 10^{-22}$, respectively by Kruskal-Wallis test). Table 2 shows the $P$-values for the pairwise Wilcoxon's rank-sum test, evaluating different levels of UPY among different stages (A), and grades (B) and the $P$ values (on the bottom) for the overall Kruskall-Wallis test, taking control samples as reference. In particular, a statistically significant difference was observed through UPY-A at early stages between Ta and T1 ( $P=0.008$ by Wilcoxon's rank-sum test) (see also Figures 2A). The sensitivity and specificity of the assay at various stages and grades are displayed in Table 3 , with the specificity being fixed at $78.82 \%$ to facilitate the comparison of the sensitivities. Consistently with the results shown in Figure 2, the sensitivity of the assay displayed striking increases from $\mathrm{Ta}$ to $\mathrm{T} 1$ or $\mathrm{T} 2-3$ (from 69.81 to $95.00 \%$ or $93.33 \%$ ) and a less pronounced increase from G1 to G2 (from 68.97 to $79.31 \%$ ). A remarkable increase of sensitivity was also observed from G2 to G3 (from 79.31 to $90.00 \%$ ). With fixed specificity at $90 \%$ (cutoff value: $335.57 \mathrm{SU}$ ), the sensitivities were $50.94 \%$ for Ta and $80.00 \%$ for T1 and T2-3. By increasing the cutoff value to 373.39 SU (thus leading specificity to $95.29 \%$ ), a sensitivity decrease was observed, especially for the earlier stages of BC, but the observed values were still above $47.17 \%$ and $75.00 \%$ in $\mathrm{Ta}$ and $\mathrm{T} 1$, respectively. We also performed the analysis of UPY levels in patients classified according to the WHO 2004 classification of BC and we observed a statistically significant difference between the low- and highgrade patients as shown in Figure $3(P=0.0005$ by Wilcoxon's rank-sum test). Moreover, a statistically significant difference was found in the levels of UPY in groups of patients with different risk of recurrence ( $P=0.002$ by Kruskal-Wallis test) (Figure $4 \mathrm{~A})$ and progression ( $P=0.001$ by Kruskal-Wallis test) (Figure $4 \mathrm{~B})$. In particular, UPY levels in the very low/low recurrence risk groups were lower compared with those in the moderate risk group ( $P=0.004$ and $P=0.002$, respectively, by Wilcoxon's rank-sum test), whereas levels of UPY in the very low progression risk group were lower compared with those in moderate risk group $(P=0.001$ by Wilcoxon's rank-sum test), strengthening the biological plausibility of the association and indicating that UPY-A could help in the prediction of progression and recurrence of BC. The increase in the prediction of recurrence and progression given by the UPY-A marker was also evaluated. The AUC of the model including EORTC risk class as predictor (model 1) was 0.61 (0.450.76); the AUC of the model including UPY-A marker as predictor (model 2) was 0.69 (0.54-0.83); finally, the AUC of the model including both variables (model 3) was $0.70(0.56-0.84)$. The increase in prediction performance was evaluated by the De Long test (model $2 v s$ model $1 P=0.30$; model 3 vs model 1 $P=0.21)$.

Effect of possible confounding factors on the diagnostic performances of UPY-A. To assess the effect of possible confounding factors, we compared UPY levels in the healthy control group stratified for age, smoking status and gender (data are shown in Supplementary Figure S1). The differences in smoking status ( $P=0.51$ by Kruskal-Wallis test) and gender ( $P=0.35$ by Wilcoxon's rank-sum test) were not statistically significant. On the contrary, after comparing the group of healthy controls older than 55 years (mean $168.6 \pm 109.4 \mathrm{SU}$ ) with those younger than 55 years (mean $134.3 \pm 123.0 \mathrm{SU}$ ), age-related differences were statistically significant $(P=0.017$ by Wilcoxon's rank-sum test), with lower UPY levels in the younger group. Odds ratios (ORs) and 95\% CIs were calculated by logistic regression for potential confounders. A statistically significant increased risk for BC among subjects presenting the UPY-A $\geqslant 261.26 \mathrm{SU}$ was observed $(\mathrm{OR}=15.30 ; 95 \% \mathrm{CI}=8.12-28.82)$. Odds ratio was still significant after the adjustment for age, smoking status and gender. Since among healthy controls the subjects older than 55 years were significantly different for UPY-A compared with younger controls, healthy controls and BC patients were stratified in four additional 
A

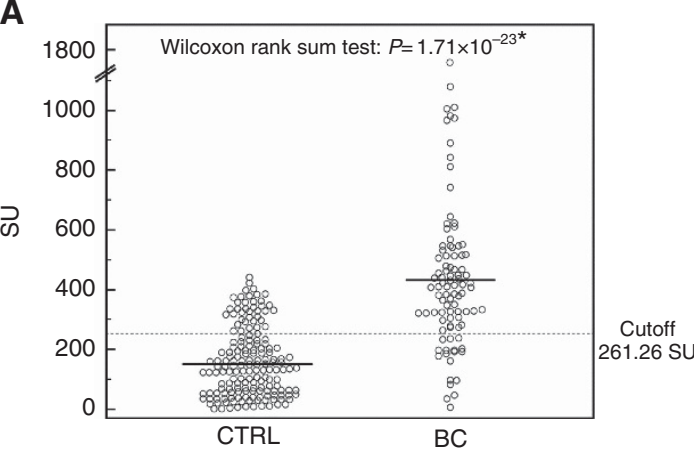

B

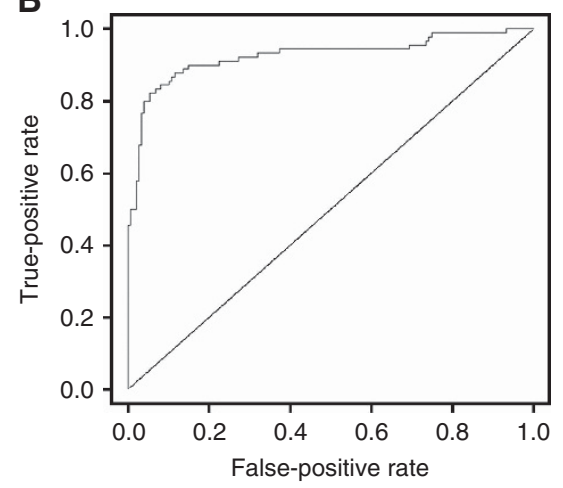

C

\begin{tabular}{lccc} 
C & OR & $\mathrm{Cl}$ & $P$-value \\
\hline UPY (SU) & 1.109 & $(1.074-1.146)$ & $3.29 \times 10^{-10}$ \\
Age & 1.040 & $(0.993-1.089)$ & 0.096 \\
Gender (females vs. males) & 0.414 & $(0.108-1.591)$ & 0.199 \\
Smoke (former vs. never) & 5.577 & $(1.514-20.543)$ & 0.010 \\
Smoke (current vs. never) & 10.352 & $(4.184-25.610)$ & $4.26 \times 10^{-7}$
\end{tabular}

Figure 1. Urinary tyrosine-phosphorylated protein levels in urine samples. (A) Analysis of urinary UPY levels in samples of healthy subjects $(n=170)$ and BC patients $(n=92)$ using the UPY-A. Healthy subject (control (CTRL)) mean levels: $157.9 \pm 114.5$ SU; BC mean levels:

$434.8 \pm 258.4$ SU. Significance of the differences: $P=1.71 \times 10^{-23}$ by Wilcoxon's rank-sum test. The solid lines indicate the mean values; the dotted line indicates the best cutoff value. (B) ROC curve of total UPY levels adjusted for age, smoking status and gender. (C) Estimated risks of BC: results of the regression model including UPY-A, age, smoking status and gender as predictors.

A

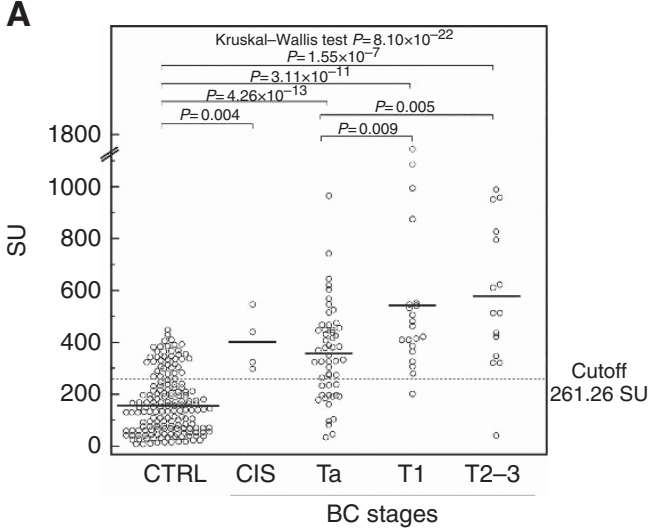

B

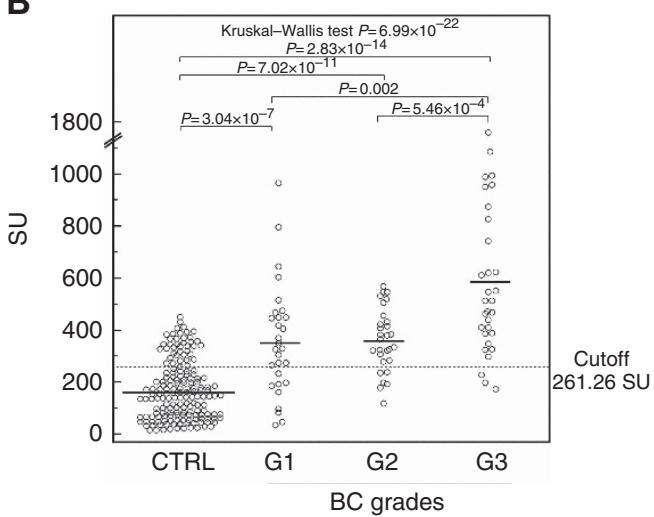

Figure 2. Urinary tyrosine-phosphorylated protein levels in subjects with different BC stages and grades. (A) Distribution of UPY levels in subjects with different BC stages. Control (CTRL), $n=170$, mean 157.9 $\pm 114.5 \mathrm{SU}$; carcinoma in situ (CIS), $n=4$, mean 400.8 $\pm 114.8 \mathrm{SU}$; Ta, $n=53$, mean $356.3 \pm 181.0 \mathrm{SU} ; \mathrm{T} 1, n=20$, mean $540.9 \pm 346.6 \mathrm{SU} ;$ and T2-3, $n=15$, mean $579.5 \pm 290.6 \mathrm{SU}$. Urinary tyrosine-phosphorylated protein levels are significantly different in groups of BC patients with different BC stages (Kruskal-Wallis test, $P=8.10 \times 10^{-22}$ ). (B) Distribution of UPY levels in subjects with different $B C$ grades. Control, $n=170$, mean 157.9 $\pm 114.5 \mathrm{SU} ; \mathrm{G} 1, n=29$, mean 357.6 $\pm 217.9 \mathrm{SU} ; \mathrm{G} 2, n=29$, mean 361.7 $\pm 122.9 \mathrm{SU}$; and $\mathrm{G} 3, n=30$, mean $584.5 \pm 336.4 \mathrm{SU}$. Urinary tyrosine-phosphorylated protein levels are significantly different in groups of BC patients with different BC grades (Kruskal-Wallis test, $P=6.99 \times 10^{-22}$ ). The solid lines indicate the mean values; the dotted line indicates the best cutoff value.

Table 2. P-values for the pairwise Wilcoxon's rank-sum test, evaluating different levels of UPY among different stages (A) and grades (B)

\begin{tabular}{l|l|c|c|c|c|c|c|c|c|c|}
\hline A & CTRL & CIS & Ta & T1 & T2-3 & B & CTRL & G1 & G2 & G3 \\
\hline CTRL & - & 0.004 & $4.26 \times 10^{-13}$ & $3.11 \times 10^{-11}$ & $1.55 \times 10^{-7}$ & CTRL & - & $3.04 \times 10^{-7}$ & $7.02 \times 10^{-11}$ & $2.83 \times 10^{-14}$ \\
\hline $\mathrm{CIS}$ & - & - & 0.532 & 0.439 & 0.230 & $\mathrm{G} 1$ & - & - & 0.592 & 0.002 \\
\hline $\mathrm{Ta}$ & - & - & - & 0.009 & 0.005 & $\mathrm{G} 2$ & - & - & - & 0.001 \\
\hline $\mathrm{T} 1$ & - & - & - & - & 0.368 & $\mathrm{G} 3$ & - & - & - & - \\
\hline $\mathrm{T} 2-3$ & - & - & - & - & - & & & & \\
\hline \multicolumn{8}{|l|}{$\mathrm{P}=8.10 \times 10^{-22}$} \\
\hline
\end{tabular}


Table 3. Diagnostic performances of UPY-A at different BC stages and grades

\begin{tabular}{|l|l|c|c|c|c|}
\hline $\begin{array}{l}\text { BC stage } \\
\text { or grade }\end{array}$ & AUC & $\begin{array}{c}\text { 95\% Confidence } \\
\text { interval }\end{array}$ & $\begin{array}{c}\text { Sensitivity (specificity 78.82\%) } \\
\text { (cut-off value 261.26 SU) }\end{array}$ & $\begin{array}{c}\text { Sensitivity (specificity 90.00\%) } \\
\text { (cut-off value 335.57 SU) }\end{array}$ & $\begin{array}{c}\text { Sensitivity (specificity 95.29\%) } \\
\text { (cut-off value 373.39 SU) }\end{array}$ \\
\hline CIS & 0.924 & $0.873-0.958$ & $100 \%$ & $50.00 \%$ & $50.00 \%$ \\
\hline Ta & 0.830 & $0.774-0.877$ & $69.81 \%$ & $50.94 \%$ & $47.17 \%$ \\
\hline T1 & 0.954 & $0.914-0.979$ & $95.00 \%$ & $80.00 \%$ & $75.00 \%$ \\
\hline T2-3 & 0.909 & $0.858-0.946$ & $93.33 \%$ & $80.00 \%$ & $73.33 \%$ \\
\hline G1 & 0.798 & $0.735-0.851$ & $68.97 \%$ & $48.28 \%$ & $41.38 \%$ \\
\hline G2 & 0.879 & $0.826-0.921$ & $79.31 \%$ & $51.72 \%$ & $48.28 \%$ \\
\hline G3 & 0.936 & $0.892-0.966$ & $90.00 \%$ & $86.67 \%$ & $83.33 \%$ \\
\hline Abbreviations: AUC = area under ROC curve; BC= bladder cancer; CIS= carcinoma in situ; UPY-A = urinary tyrosine-phosphorylated protein assay. \\
\hline
\end{tabular}

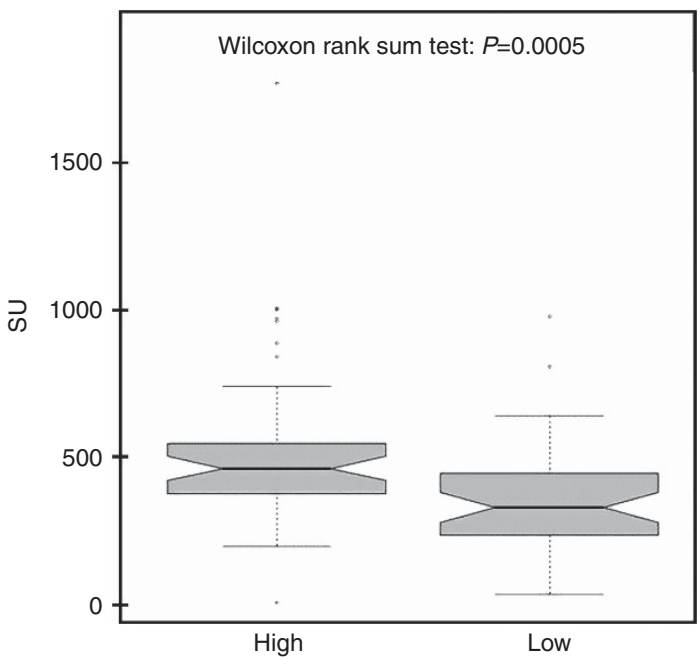

Figure 3. Urinary tyrosine-phosphorylated protein levels in BC subjects classified according to the WHO 2004 classification of BC. High grade, $n=43$, mean $528.06 \pm 297.19 \mathrm{SU}$; low grade, $n=43$, mean $354.22 \pm 191.16 \mathrm{SU}$. Urinary tyrosine-phosphorylated protein levels are significantly different in groups of $\mathrm{BC}$ patients with different $\mathrm{BC}$ grade (Kruskal-Wallis test, $P=0.0005$ ).

different age groups. Figure 5A shows that UPY levels are significantly increased in BC patients $v s$ healthy controls in all age groups. The figure also highlights that healthy controls under 55 years display lower UPY levels with respect to older controls, not showing reciprocal variations. On the other hand, BC patients showed a progressive increase of UPY with the age because of the increasing prevalence of more advanced tumour stages in older patients. In patients under 55 years, we observed a large prevalence of Ta and G1 (Figures 5B and C). As a matter of fact, the best cutoff limit in this age group was $180 \mathrm{SU}$ instead of $261.26 \mathrm{SU}$. With this cutoff limit, sensitivity improved from 54.5 to $81.8 \%$.

\section{DISCUSSION}

Changes of protein tyrosine-phosphorylation are involved in cell growth and differentiation and have been observed in many cancer types, usually as a consequence of altered tyrosine kinase activity (Blume-Jensen and Hunter, 2001; Lim, 2005; Harsha and Pandey, 2010). On the other hand, tyrosine kinases are among the most important oncogenes known to date, as they have a central role in cancer development and progression (Hunter, 1998; Blume-Jensen and Hunter, 2001; Lim, 2005). Robust evidence demonstrates the involvement of abnormal kinase activity in $\mathrm{BC}$ following mutations and/or overexpression of protein kinases ( $\mathrm{Al}$ Hussain and Akhtar, 2013), protein hyperphosphorylation in biopsy specimens (Khadjavi et al, 2011) and after using tyrosine kinase inhibitors for BC treatment (Mitra et al, 2006; Wallerand et al, 2010). Fluorescence in situ hybridsation analysis of Aurora kinase A has been used as a marker for BC (Park et al, 2008).

Measuring the effects of abnormal protein kinase activity on protein phosphorylation takes advantage from a substantial amplification of the signal, as a consequence of increased catalytic activity of the mutated kinase. However, the instability of phosphoproteins in blood has limited their use for cancer diagnosis. On the contrary, we previously observed that protein phosphatases activity is negligible in urine, thus conferring a particular stability to urinary phosphoproteins (Khadjavi et al, 2011). Nevertheless, only a small amount of phosphoproteins is associated with BC; therefore, stringent purification steps and high sensitivity detection methods are required (Khadjavi et al, 2011, 2013).

The present report evaluates and optimises the performances of the UPY examined in the pilot study (Khadjavi et al, 2013) by confirming the high sensitivity and specificity of the assay to detect BC in an independent and larger cohort of subjects. A statistically significant difference was found in the levels of UPY at different BC stages and grades. Sensitivity values have been measured at different fixed specificities. As shown in Table 3, UPY-A with chosen specificity comparable to cytology (>93\%) displays higher sensitivity in $\mathrm{Ta}(47 \%$ vs $26 \%)$ and $\mathrm{T} 1$ (74\% vs 64\%), respectively, and more than two-fold higher sensitivity in G1 subjects (41\% vs 6\%) (for the cytology data see Saad et al, 2001). Nevertheless, the comparison with other techniques is very complex, and larger and independent studies are certainly needed. The comparison should also consider additional characteristics such as the cost, the productivity and the intra/interlaboratory standardisation of the test. With regard to this, UPY-A will be in the cost range of an ELISA test such as BTA and NMP22 tests with additional advantages as UPY-A high throughput and the possibility of automated calibration in each analytical sessions, making it simpler to standardise compared with techniques requiring larger effort for interlaboratory harmonisation (Behrens et al, 2014).

In the present report, we have also observed that UPY-A can identify patients more prone to recurrence and progression. Therefore, these patients could receive closer surveillance or more aggressive therapy. Of note, the increase in the prediction performance in predicting recurrence and progression given by the UPY-A with respect to the EORTC risk class is not statistically significant, but the association of the UPY-A and the EORTC classification improves the recurrence and progression prediction. We also have excluded that age, smoking status and gender can affect the diagnostic performances of the assay as confounding factors. Nevertheless, taking into account that the average values of the test were lower in younger healthy subjects ( $\leqslant 55$ years old), we have found that lowering the cutoff limit in this group of subjects determined a significant improvement of sensitivity. Therefore, these results encourage further studies involving a wider number of 

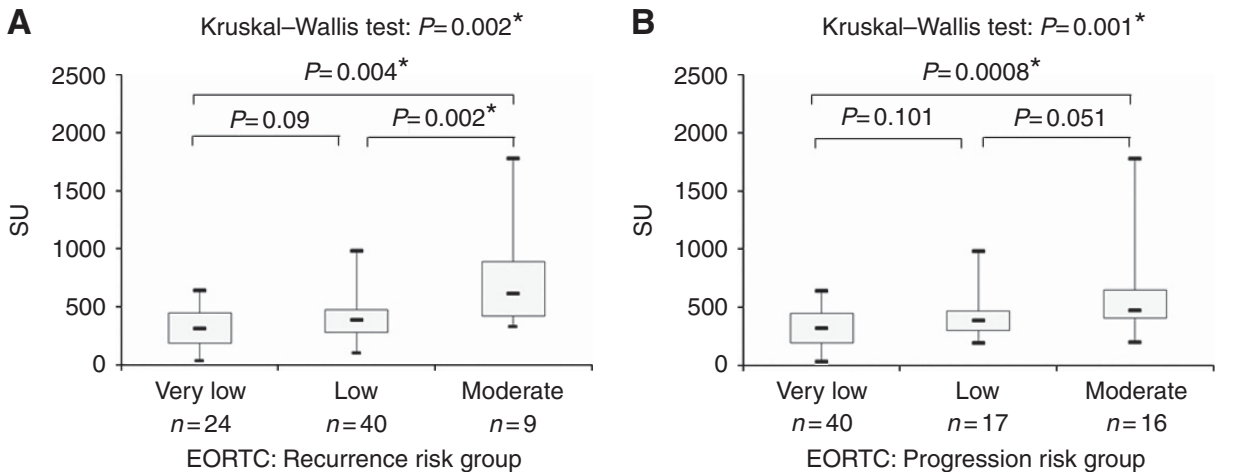

Figure 4. Urinary tyrosine-phosphorylated protein levels in relation to classification of recurrence and progression risks. (A) Analysis of UPY levels in relation to classification of recurrence risks of $B C$ patients (Kruskal-Wallis test, $P=0.002$ ). (B) Analysis of UPY levels in relation to classification of progression risk of $B C$ patients (Kruskal-Wallis test, $P=0.001$ ).

A

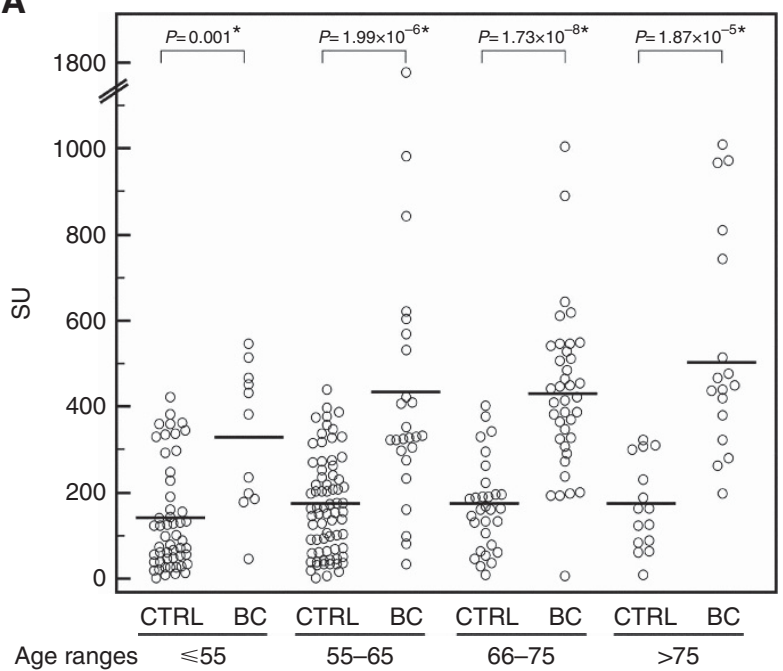

B

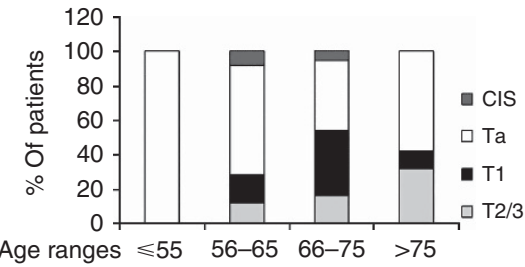

C

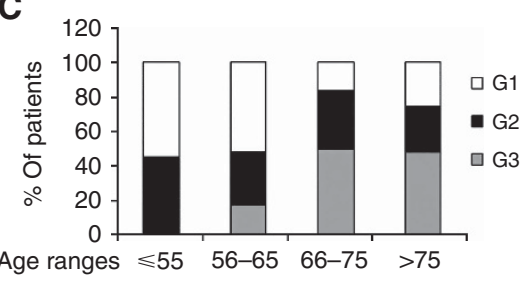

Figure 5. Urinary tyrosine-phosphorylated protein levels in subjects with different age ranges. (A) Distribution of UPY levels in control (CTRL) and $B C$ patients with age ranges: $\leqslant 55$ years old (CTRL, $n=53$, mean 134.3 $\pm 123.0 \mathrm{SU} ; \mathrm{BC}, n=11$, mean 326.8 $\pm 164.9 \mathrm{SU}), 56-65$ years old (CTRL, $n=72$, mean 169.2 $\pm 114.0 \mathrm{SU} ; \mathrm{BC}, n=25$, mean 437.2 $\pm 354.5 \mathrm{SU}), 66-75$ years old (CTRL, $n=28$, mean 168.5 $\pm 108.0 \mathrm{SU}$; $B C$, $n=37$, mean $431.2 \pm 187.1 \mathrm{SU}$ ) and $>75$ years old (CTRL, $n=15$, mean 168.2 $\pm 103.4 \mathrm{SU} ; \mathrm{BC}, n=19$, mean $501.0 \pm 270.6 \mathrm{SU}$ ). Urinary tyrosine-phosphorylated protein levels are significantly increased in BC patients in all age groups (Wilcoxon's rank-sum test). The solid lines indicate the mean values. (B) Distribution of patients with different BC stages: data are expressed as percentage in different age groups. (C) Distribution of patients with different $B C$ grades: data are expressed as percentage in different age groups.

patients younger than 55 year, to optimise the cutoff values. Interestingly, a first preliminary investigation on a group of patients with nonmalignant urological disorders did not reveal considerable interference. Large and independent studies are currently in progress to confirm the present data and to evaluate the value of UPY-A in the follow-up of BC patients.

In conclusion, the present study supplies important information on the diagnostic characteristics of UPY-A revealing remarkable performances for early stages. The efforts made to miniaturise the method markedly increased its throughput allowing its potential use for a wide range of applications encompassing the screening of high-risk subjects, primary diagnosis and posttreatment surveillance.

\section{ACKNOWLEDGEMENTS}

We thank Milena Maria Maule and Federica Di Nicolantonio for their comments and suggestions. We also thank the Molinette Hospital Blood Bank and Urology Day Surgery personnel for help with the urine collection. This study was supported by the PIA2010, Regione Sardegna (Italy) to FM and FT.

\section{REFERENCES}

Al Hussain TO, Akhtar M (2013) Molecular basis of urinary bladder cancer. Adv Anat Pathol 20: 53-60.

Babjuk M, Böhle A, Burger M, Compérat E, Kaasinen E, Palou J, van Rhijn BWG, Rouprêt M, Shariat S, Sylvester R, Zigeuner R (2014) Guidelines on non-muscle-invasive bladder cancer (Ta, T1 and CIS). Eur Assoc Urol 2014: $1-48$

Behrens T, Bonberg N, Casjens S, Pesch B, Brüning T (2014) A practical guide to epidemiological practice and standards in the identification and validation of diagnostic markers using a bladder cancer example. Biochim Biophys Acta 1844: 145-155.

Blume-Jensen P, Hunter T (2001) Oncogenic kinase signaling. Nature 411: 355-365.

Boman H, Hedelin H, Jacobsson S, Holmäng S (2002) Newly diagnosed bladder cancer: the relationship of initial symptoms, degree of microhematuria and tumor marker status. J Urol 168: 1955-1959. 
Chavan S, Bray F, Lortet-Tieulent J, Goodman M, Jemal A (2014) International variations in bladder cancer incidence and mortality. Eur Urol 66: 59-73.

Cheung G, Sahai A, Billia M, Dasgupta P, Khan MS (2013) Recent advances in the diagnosis and treatment of bladder cancer. BMC Med 11: 13.

DeLong ER, DeLong DM, Clarke-Pearson DL (1988) Comparing the areas under two or more correlated receiver operating characteristic curves: a nonparametric approach. Biometrics 44: 837-845.

Gore JL, Gilbert SM (2013) Improving bladder cancer patient care: a pharmacoeconomic perspective. Expert Rev Anticancer Ther 13: 661-668.

Greene KL, Albertsen PC, Babaian RJ, Carter HB, Gann PH, Han M, Kuban DA, Sartor AO, Stanford JL, Zietman A, Carroll P (2013) Prostate specific antigen best practice statement: 2009 update. J Urol 189: S2-S11.

Harsha HC, Pandey A (2010) Phosphoproteomics in cancer. Mol Oncol 4: 482-495.

Hong YM, Loughlin KR (2008) Economic impact of tumor markers in bladder cancer surveillance. Urology 71: 131-135.

Hunter T (1998) The role of tyrosine phosphorylation in cell growth and disease. Harvey Lect 94: 81-119.

Khadjavi A, Barbero G, Destefanis P, Mandili G, Giribaldi G, Mannu F, Pantaleo A, Ceruti C, Bosio A, Rolle L, Turrini F, Fontana D (2011) Evidence of abnormal tyrosine phosphorylated proteins in the urine of patients with bladder cancer: the road toward a new diagnostic tool? J Urol 185: 1922-1929.

Khadjavi A, Notarpietro A, Mannu F, Pantaleo A, Ferru E, Destefanis P, Fontana D, Turrini F (2013) A high-throughput assay for the detection of Tyr-phosphorylated proteins in urine of bladder cancer patients. Biochim Biophys Acta 1830: 3664-3669.

Kriegmair M, Baumgartner R, Knüchel R, Stepp H, Hofstädter F, Hofstetter A (1996) Detection of early bladder cancer by 5 -aminolevulinic acid induced porphyrin fluorescence. J Urol 155: 105-109; discussion 9-10.

Lim Y (2005) Mining the tumor phosphoproteome for cancer markers. Clin Cancer Res 11: 3163-3169.

Miremami J, Kyprianou N (2014) The promise of novel molecular markers in bladder cancer. Int J Mol Sci 15: 23897-23908.

Mitra A, Datar R, Cote R (2006) Molecular pathways in invasive bladder cancer: new insights into mechanisms, progression, and target identification. J Clin Oncol 24: 5552-5564.
Park HS, Park WS, Bondaruk J, Tanaka N, Katayama H, Lee S, Spiess PE, Steinberg JR, Wang Z, Katz RL, Dinney C, Elias KJ, Lotan Y, Naeem RC, Baggerly K, Sen S, Grossman HB, Czerniak B (2008) Quantitation of Aurora kinase A gene copy number in urine sediments and bladder cancer detection. J Natl Cancer Inst 100: 1401-1411.

Parker J, Spiess PE (2011) Current and emerging bladder cancer urinary biomarkers. ScientificWorldJournal 11: 1103-1112.

Ploeg M, Aben KK, Kiemeney LA (2009) The present and future burden of urinary bladder cancer in the world. World J Urol 27: 289-293.

Saad A, Hanbury DC, McNicholas TA, Boustead GB, Woodman AC (2001) The early detection and diagnosis of bladder cancer: a critical review of the options. Eur Urol 39: 619-633.

Sarosdy MF, Schellhammer P, Bokinsky G, Kahn P, Chao R, Yore L, Zadra J, Burzon D, Osher G, Bridge JA, Anderson S, Johansson SL, Lieber M, Soloway M, Flom K (2002) Clinical evaluation of a multi-target fluorescent in situ hybridization assay for detection of bladder cancer. J Urol 168: 1950-1954.

Schneeweiss S, Kriegmair M, Stepp H (1999) Is everything all right if nothing seems wrong? A simple method of assessing the diagnostic value of endoscopic procedures when a gold standard is absent. J Urol 161: 1116-1119.

Simon MA, Lokeshwar VB, Soloway MS (2003) Current bladder cancer tests: unnecessary or beneficial? Crit Rev Oncol Hematol 47: 91-107.

Wallerand H, Robert G, Bernhard JC, Ravaud A, Patard JJ (2010) Tyrosinekinase inhibitors in the treatment of muscle invasive bladder cancer and hormone refractory prostate cancer. Arch Esp Urol 63: 773-787.

Zaak D, Kriegmair M, Stepp H, Baumgartner R, Oberneder R, Schneede P, Corvin S, Frimberger D, Knüchel R, Hofstetter A (2001) Endoscopic detection of transitional cell carcinoma with 5-aminolevulinic acid: results of 1012 fluorescence endoscopies. Urology 57: 690-694.

This work is published under the standard license to publish agreement. After 12 months the work will become freely available and the license terms will switch to a Creative Commons AttributionNonCommercial-Share Alike 4.0 Unported License.

Supplementary Information accompanies this paper on British Journal of Cancer website (http://www.nature.com/bjc) 